Gut, 1971, 12, 427-431

\title{
Ileo-rectal anastomosis for Crohn's disease of the colon
}

\author{
W. N. W. BAKER \\ From the Research Department, St Mark's Hospital, London
}

SUMmary Twenty-six cases of Crohn's disease of the colon treated by total colectomy and ileorectal anastomosis have been examined. Recurrent disease at the anastomosis occurred in $50 \%$ of patients, three-quarters of these within 18 months and the latest six years after operation. Patients with disease apparent only in the colon at the primary operation developed ileal recurrence at the anastomosis as commonly as those with both ileum and colon involved initially. Recurrence in the small intestine away from the anastomosis was uncommon. The response of recurrent disease to medical treatment was encouraging and further surgical intervention has been avoided in the majority of cases.

Complicated anal fistulae developed in $20 \%$ of patients up to seven years after ileo-rectal anastomosis. They materially affected the management of the individual patient but most responded to conventional surgical treatment after a prolonged healing period.

Ileo-rectal anastomosis for Crohn's disease of the colon has had a lower operative morbidity than the same operation performed for ulcerative colitis, but the long-term prognosis has been worse because of the development of recurrent disease and serious anal lesions.

No reports have yet been published dealing exclusively with the results of total colectomy and ileorectal anastomosis for Crohn's disease of the colon. The operation is mentioned in most surveys of the surgical treatment of Crohn's disease of the colon but has not been differentiated from other ileo-colic, or colo-rectal anastomoses.

The object of this paper is to assess the results of treatment of Crohn's disease by this surgical procedure, compare them with the results of ileo-rectal anastomosis for ulcerative colitis, and to examine two problems particularly associated with Crohn's disease, namely, intestinal recurrences and anal lesions.

\section{Material}

All patients undergoing ileo-rectal anastomosis for inflammatory disease of the colon at St Mark's Hospital from 1953 to 1969 have been reviewed and divided into the following groups on a pathological basis: (1) ulcerative colitis, (2) Crohn's disease, and (3) colitis unclassified.

The problems of making the diagnosis of Crohn's Received for publication 19 April 1971. disease of the colon have been dealt with by other authors (Lockhart-Mummery and Morson, 1964; Janowitz, Lindner, and Marshak, 1965; LennardJones, Lockhart-Mummery, and Morson, 1969), and any patient in this series who did not meet the histological criteria for Crohn's disease or ulcerative colitis was placed in a separate category of 'colitis unclassified'. This paper deals only with the results of patients classified as having Crohn's disease. The ulcerative colitis patients were reviewed in a previous publication (Baker, 1970), and the "colitis unclassified' patients have been excluded from both surveys.

Twenty-eight patients with Crohn's disease of the colon have been treated by colectomy and ileo-rectal anastomosis during this period. The main criterion for selection of the patients preoperatively was the presence of a normal, or near normal, rectum. There were 16 men and 12 women, with an age range at the time of operation of from 18 to 65 years.

All but three of the surviving patients were followed up by interview and physical examination in the outpatient department. The three patients who were unable to attend were followed up by letter; two of them failed to reply, leaving 26 patients for study. 
The length of follow up extended from six months to 16 years with all surviving patients having been followed up for a minimum of one year after operation. The mean follow-up time was six years two months with a median of five years.

\section{Results of Surgical Treatment}

These have been divided into 'good', 'fair', and 'poor', according to the following criteria:

GOOD

Survival following operation for at least one year with no further treatment required for Crohn's disease or other complications up to the time of follow up.

FAIR

Survival following operation for at least one year but still requiring treatment for Crohn's disease to maintain good health.

POOR

Patients who have died from Crohn's disease or have had to be converted to a permanent ileostomy.

There are relatively few good results $(5 / 26)$ compared with fair ones (14/26), and nearly a quarter of the patients $(7 / 26)$ have been classified as poor.

\section{Reasons for Poor Results}

Two patients were classified as operative failures: one operative death from intraperitoneal haemorrhage, and one anastomotic leak leading to excision of the rectum and a permanent ileostomy. The other five patients had a poor result because of the later progression of the Crohn's disease. Two died, one from malnutrition, chronic sepsis, and amyloid disease, and the other from peritonitis secondary to necrosis of the rectum three months after discharge from hospital. The other three patients had to have the rectum excised and a permanent ileostomy established for severe, persistent rectal disease. These results are set out in Table I.

\begin{tabular}{ll}
\hline Operative & \\
$\quad$ Haemorrhage (died) & 1 \\
Anastomotic leak & 1 \\
Excision of rectum for active Crohn's disease & \\
$\quad$ Malnutrition, chronic sepsis (died) & 1 \\
Late rectal necrosis (died) & 1 \\
$\quad$ Severe disease & 3 \\
Other deaths & \\
$\quad$ Carcinomatosis & 1 \\
Hodgkin's disease & 1 \\
Unknown & 1 \\
\hline
\end{tabular}

Table I Reasons for poor results and deaths

\section{Deaths}

Six patients have died. The three deaths due to operation and persistent Crohn's disease have been mentioned above. Two other deaths were from malignant diseases: one from carcinomatosis with a primary in the colon at the time of ileo-rectal anastomosis, and the other from Hodgkin's disease. The sixth patient is known to have died some years after operation but no information is available about the cause.

\section{Operative Morbidity}

The operative morbidity in this series was low. There was one major anastomotic leak leading to peritonitis and pelvic sepsis, and one other patient had a repeat ileo-rectal anastomosis for stricturing at the anastomosis not associated with Crohn's disease.

\section{Recurrent Disease}

The term 'recurrent disease' used here includes both exacerbations of disease in areas of bowel known to have been diseased before operation, and the appearance of active inflammation, with the radiological or histological characteristics of Crohn's disease in parts of the bowel thought previously to be free of involvement.

The detailed pre- and postoperative information necessary for a study of recurrent disease was available for 25 patients. The findings are summarized in Figure 1.

Only four patients were apparently free of disease at follow up times of 16,11 , three, and one years respectively. Recurrence at the anastomosis occurred in 13 patients. Disease on both ileal and rectal sides of the anastomosis was the commonest finding in 11 patients; the other two patients showed evidence of disease on the rectal side of the anastomosis only. Seven patients had active disease in the distal rectum only, away from the anastomosis. Three patients developed disease in the proximal ileum as well as on both sides of the anastomosis, and one patient had a recurrence in the proximal ileum only.

The distribution of the disease at the time of the initial operation was judged on radiological evidence, clinical examination of the bowel at operation, and histological examination of the specimen. On this evidence the patients were divided into (1) patients with disease apparently confined to the colon; (2) patients with disease manifest in both colon and ileum.

The site of recurrence was analysed according to the distribution of the disease at the time of the initial operation (Table II). This showed that the site of 


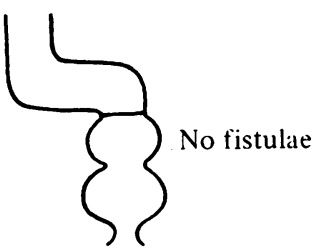

No recurrence (4)

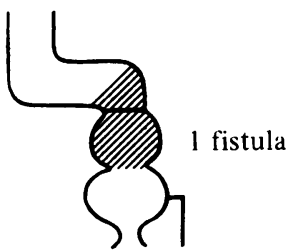

Anastomosis only (2)

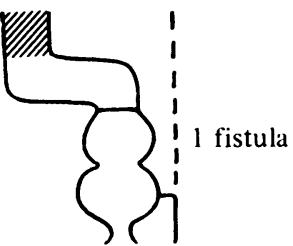

Prox. ileum only (1)

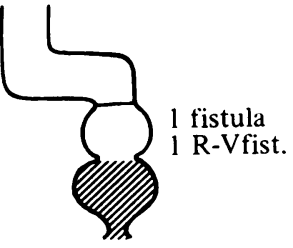

Lower rectum only (7)

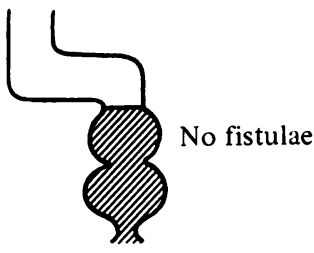

Rectum up to anastomosis (2). recurrence was not apparently influenced by the preoperative distribution. It was significant that patients with the disease apparently confined to the colon at the time of operation were not immune from recurrence in the ileum. Of the 10 patients thought to have colonic disease only at operation, five subsequently developed ileal disease, all at the anastomosis and two in the proximal ileum as well.

\begin{tabular}{lll}
\hline Sites of Recurrence & Distribution at Operation \\
\cline { 2 - 3 } & Colon Only (10) $\begin{array}{l}\text { Colon and Ileum } \\
(15)\end{array}$ \\
\hline None & 2 & 2 \\
Anastomosis, ileum and rectum & 5 & 6 \\
Anastomosis, rectum only & 0 & 2 \\
Distant ileum & 2 & 2 \\
Distal rectum (not anastomosis) & 3 & 4
\end{tabular}

Table II Disease distribution at initial operation related to site of recurrence

${ }^{1}$ The totals do not correspond with the number of patients because of recurrence both in the distant ileum and anastomosis in three patients.

\section{Anal Lesions}

Anal lesions have been classified into two groups: (1) minor lesions which responded to conventional treatment and did not significantly influence the course of the disease; (2) serious lesions which radically altered the clinical picture and influenced decisions about major surgical treatment.
The first group included skin tags, fissures, abscesses, low fistulae, and simple ulcers. These were treated successfully by conventional methods. Most of them occurred after ileo-rectal anastomosis but several were treated preoperatively and showed no particular tendency to recur. The second group consisted of complicated fistulae.

All fistulae occurring both as serious and minor lesions were documented in detail, and Table III tabulates the individual lesions in relation to their time of appearance and response to treatment. For the sake of simplicity they were classified as simple (S) if their tracks remained below the external sphincter, and complicated (C) if their tracks involved the external sphincter or pierced the levator ani muscle.

The results showed that a simple fistula treated before or at the time of operation healed or caused little trouble. The fistulae occurring after ileo-rectal anastomosis were all of the complicated type but they too responded to conventional treatment in most cases although the healing time was prolonged. So far only one has been a major factor in the decision to remove the rectum. In the fistulae associated with active Crohn's disease of the rectum healing time was prolonged but still occurred if the rectal disease was controlled by medical therapy directed at the Crohn's disease. Figure 1 shows the fistulae occurring after ileo-rectal anastomosis and their relationship to recurrent disease. Table III 


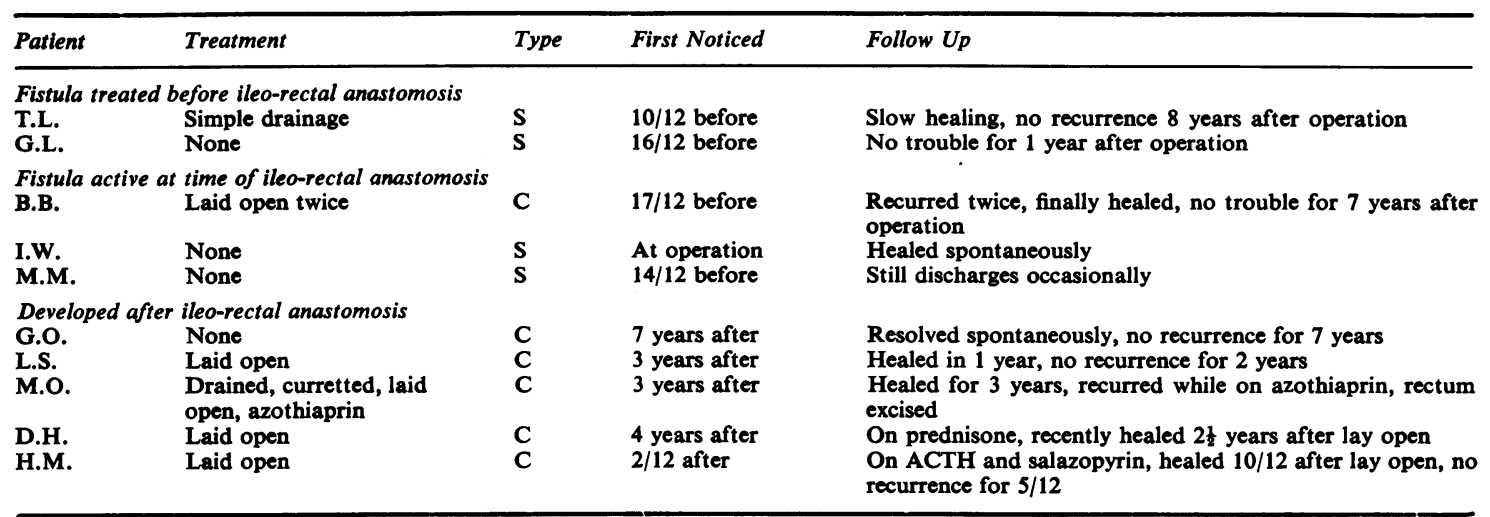

Table III Detailed results of treatment of fistulae

$\mathbf{S}=$ simple fistula, $\mathbf{C}=$ complicated fistula.

shows that complicated fistulae have developed up to seven years after ileo-rectal anastomosis.

Three patients suffered from recto-vaginal fistulae. None of them received any surgical treatment directed at the lesion, and none of the fistulae healed spontaneously. Two developed after ileo-rectal anastomosis, at four months and nine years respectively, and were associated with severe rectal Crohn's disease. The other patient had a low fistula at the time of operation which is still patent and causes little inconvenience.

\section{Discussion}

COMPARISON OF RESULTS OF ILEO-RECTAL ANASTOMOSIS FOR CROHN'S DISEASE AND ULCERATIVE COLITIS

Although clinically it may be difficult to distinguish between ulcerative colitis and Crohn's disease of the colon, particularly in the acute phase, in the long term the differences between the two diseases become more obvious. When the present series was compared with a similar group of patients undergoing ileorectal anastomosis for ulcerative colitis at St Mark's Hospital (Baker, 1970), it became apparent that the definitions of 'good' or 'fair' success and 'failure' used for ulcerative colitis cases were not readily applicable to those of Crohn's disease. The chronicity of Crohn's disease and its tendency to recur make any claim to 'success', implying a permanent cure, inappropriate. It might have been better to classify Crohn's disease in the same way as malignant disease and refer to five- and 10-year cures, but for the sake of simplicity a clinical classification, based on the state of the patient at the time of follow up of 'good', 'fair', and 'poor', was chosen. Bearing in mind the limitations of classification some valid comparisons between the results for the two diseases can still be made. About $35 \%$ of the ulcerative colitis cases were classed as 'good successes', and these may be compared with the $20 \%$ 'good' results of the Crohn's patients. There were $20 \%$ 'fair successes' among the ulcerative colitis patients, and these correspond to the $50 \%$ 'fair' results of the Crohn's patients. Likewise the $50 \%$ 'failures' in the ulcerative colitis series contrast with the $25 \%$ 'poor' results in the Crohn's series. Another point worth noting was the marked difference in the number of instances of anastomotic leakage and peri-operative sepsis between the two series; nine ulcerative colitis patients suffered these complications compared with only one Crohn's patient.

The overall incidence of anal lesions in the ulcerative colitis patients was $30 \%$ and of complicated fistulae $10 \%$. This compares with an overall incidence of $75 \%$ in the Crohn's patients with $21 \%$ complicated fistulae.

These comparisons between the two series show that the operation itself has been more hazardous in ulcerative colitis than in Crohn's disease and accompanied by a high morbidity and initial failure rate. The long-term prognosis for ulcerative colitis has been better than for Crohn's disease because of the high incidence of recurrence and serious anal lesions in the latter. The reason for these differences probably lies in the nature of the two diseases. The lower operative complication rate in Crohn's disease reflects the relative normality of the ileal and rectal tissues at the time of operation, and the poorer longterm results in Crohn's disease reflects the chronicity of the disease and its tendency to recur.

\section{RECURRENT DISEASE}

Proof of recurrent Crohn's disease, particularly of 
the small bowel, is frequently difficult to obtain. Bowel which appears normal on naked-eye or radiological appearance can show histological evidence of Crohn's disease, and bowel which looks like Crohn's disease clinically may present a non-specific picture under the microscope. In this series the clinical, radiological, and pathological evidence has been assessed for each patient in an effort to reduce this source of error but absolute proof of recurrence has not always been obtained.

The pattern of recurrence observed gives some support to the view of Korelitz (1967) that the disease appears to spread after surgical intervention. The cases he examined had been selected for operation on the basis of a normal, or near normal rectum, as had the cases in this series. His finding of a subsequent flare up in the previously uninvolved portions of rectum and ileum has been confirmed in this series.

The difficulty of assessing the extent of the disease preoperatively and the small number of patients studied makes the division of patients into groups with colonic disease only, and colon plus ileal involvement, rather artificial. However, failure to demonstrate ileal disease before operation has not lessened the chances of an ileal recurrence. Half the 'colon only' group developed an ileal recurrence postoperatively compared with a third of the patients with colon and ileum involved initially. It is also interesting to compare the incidence of recurrent disease in the ileum after ileo-rectal anastomosis with that after total proctocolectomy for Crohn's disease. There were 10 known ileal recurrences in this series out of 25 patients, whereas there have been only four ileal recurrences in 69 patients undergoing total proctocolectomy for Crohn's disease of the colon over the same period at St Mark's. Allowing for the lack of any attempt to match the two groups, the difference is sufficiently striking to provoke further investigation into this aspect of the problem.

\section{MEDICAL TREATMENT}

The response of recurrences to medical treatment has been encouraging and repeated operations have been avoided in most cases where no mechanical obstruction has been present. The mainstay of the medical regime has been the use of oral sulphonamides and steroids given orally, systemically, and locally via retention enemata. The sulphonamides and steroids have been used separately and in combination and a process of trial and error has had to be adopted to find the best treatment for each individual patient.

\section{ANAL LESIONS}

The high incidence of anal lesions associated with Crohn's disease of the colon observed by other authors (Yarnis and Crohn, 1960; LockhartMummery, and Morson, 1964) has been confirmed, and their presence has helped in making the clinical diagnosis. Minor lesions have responded well to standard treatment although healing time has often been prolonged. Their presence has not materially affected the course of the disease or decisions on major surgical procedures. Complicated fistulae have proved difficult to treat, healing time has been prolonged, and they have tended to recur. However, one high fistula in this series, which appeared after ileorectal anastomosis, healed spontaneously without surgical treatment, which emphasizes the difficulty in making an accurate prognosis in the individual patient. In general, if the ano-rectal lesion has been associated with severe rectal disease the outlook for conservative treatment has been poor.

I wish to express my thanks to the consultant staff of St Mark's Hospital for permission to examine patients under their care, and to $\mathrm{Mr} \mathrm{H}$. E. LockhartMummery, Dr J. E. Lennard-Jones, and Dr B. C. Morson, for their helpful criticism in the preparation of this paper.

\section{References}

Baker, W. N. W. (1970). The results of ileorectal anastomoses at St Mark's Hospital from 1953 to 1968. Gut, 11, 235-239.

Janowitz, H. D., Lindner, A. E., and Marshak, R. H. (1965). Granulomatous colitis: Crohn's disease of the colon. J. Amer. med. Ass., 191, 825-828.

Korelitz, B. I. (1967). Clinical course, late results, and pathological nature of inflammatory disease of the colon initially sparing the rectum. Gut, 8, 281-290.

Lennard-Jones, J. E., Lockhart-Mummery, H. E., and Morson, B. C. (1968). Clinical and pathological differentiation of Crohn's disease and proctocolitis. Gastroenterology, 54, 1162-1170.

Lockhart-Mummery, H. E., and Morson, B. C. (1964). Crohn's disease of the large intestine. Gut, 5, 493-509.

Yarnis, H., and Crohn, B. B. (1960). Segmental (ulcerative) colitis. Gastroenterology, 38, 721-728. 